\title{
Oral contraceptives and breast cancer
}

\author{
Slight increase in risk, but must be seen in the context of all forms of contraception
}

Whether hormonal contraceptives cause breast cancer is an important public health question, as well as one of great importance to women and men choosing their method of family planning. Breast cancer is common and on the increase. Use of hormonal contraceptives is also common and is expected to increase worldwide. Thus, even a small increase in the risk of breast cancer in women using oral contraceptives would be important because of the frequency of the exposure.

A systematic review recently published in the Lancet contributes to our knowledge of the relation between oral contraceptives and breast cancer. ${ }^{1}$ Results of 54 case-control studies (some of them with a nested design) were pooled to compare the past exposure to oral contraceptive pills among women with and without a history of breast cancer. Results are given overall (adjusted relative risk 1.07) and stratified by different patterns of use. The review concludes that women who currently use or have recently stopped using oral contraceptive pills have a slightly increased risk of breast cancer (odds ratio 1.24 for current users and 1.16 for women who stopped using the pill one to four years before). No increase is seen after 10 years of stopping the pill, and the breast cancers were clinically less advanced than among nonusers.

Can we trust these findings? The review was done by experienced epidemiologists, overall numbers were certainly large enough, and the data were carefully analysed and reported. But pooling data cannot overcome the limitations of individual case-control studies. The report does not tell us how the controls were chosen, nor whether they were representative of the population from which the cases came.

Some confounding factors were controlled for in the analysis, but the biases inherent in non-experimental studies are evident-especially those due to selection. Firstly, oral contraceptives are used mainly by fertile women who are having regular sexual intercourse. The risk of breast cancer in these women may be quite different from the risk in women who are not sexually active or who are infertile. Secondly, in many populations there is widespread anxiety about the risk of cancer from taking female sex hormones, ${ }^{2}$ and it is quite possible that women who believe themselves to be at high risk of breast cancer may decide not to use oral contraception. In the current study some analyses were made according to whether a woman's mother or sister had had breast cancer, but this is a crude measure of genetic risk and the information was available for only a small number of studies. Thirdly, many women stop using oral contraceptives because of side effects, and we do not know how sensitivity to external hormones is related to endogenous hormone production and risk of breast cancer.

Even if we ignore these inherent methodological problems, are the review's findings relevant to today's users of the pill?
Most of the women studied would have started the pill during the $1960 \mathrm{~s}$. The review did not find a decreased risk of breast cancer for low dose oral contraceptives, suggesting that the physiological effects of oral contraceptives are more important than the content of individual preparations. Still, the possible importance of the chemical contents of oral contraceptives in contributing to conditions other than breast cancer cannot be excluded, as illustrated by the recent discussion of oral contraceptives and the risk of thrombotic events. ${ }^{3}$

Patterns of use of oral contraceptives are complicated, and the age at starting and stopping, continuity and duration of use, and relation to pregnancies, childbearing, and breast feeding may all have an impact on the risk of breast cancer. At the time when the studies were done, prolonged use before first pregnancy was rare, and even after results were pooled the number of such women was small. The median duration of use was three years and the median age of starting the pill was 26 years. Early use of oral contraceptives and delayed first pregnancy are increasingly common in many countries, ${ }^{456}$ and use for 10 or more years before first pregnancy is not uncommon. The trend towards earlier sexual activity and efforts to prevent unintended pregnancies are likely to reinforce this pattern of use.

The review examined various patterns of use but not long term use before first pregnancy. In view of the current trend towards starting use before the age of 20 , this would have been important. Neither does the review look at potential long term consequences, when the early starters reach the age of high breast cancer risk or when users enter the new hormonal balance of menopause and possibly postmenopausal hormone therapy. ${ }^{7}$ These deficiences may well be explained by deficiencies in the original data. Studies are needed on the health effects of both long term use before first pregnancy and long term follow up of such users.

The finding of more localised cancers among users of the pill may be explained by earlier diagnosis or the influence of contraceptive hormones on tumour growth. The increased risk of breast cancer among current users and a decline in risk after stopping the pill support the theory that oral contraceptives work as tumour promoters rather than as carcinogenic factors. However, one cannot exclude the possibility of selection bias: it is possible that women who develop rapidly progressive breast cancer at an early age are those who have not needed oral contraception (due to their sexual habits or infertility) or have wanted to avoid the pill because of their family history.

What are the public health implications of this study? The researchers have attempted to translate the odds ratios into numbers of extra cancers occurring. But they could assess only the impact of short term use (up to four years) with relatively 
short term follow up. The impacts of current patterns of use must await further studies. When women and men make their sexual and reproductive choices, and when physicians give their advice, they are interested in all potential consequences, not only breast cancer. Case-control studies cannot provide data on more than one outcome, and we must therefore wait for a systematic review of cohort studies comparing all relevant health effects of oral contraceptives with those of alternative

1 Collaborative Group on Hormonal Factors in Breast Cancer. Breast cancer and hormonal contraceptives: collaborative reanalysis of individual data on 53297 women with and 100239 women without breast cancer from 54 epidemiological studies. Lancet 1996;347:1713-27.

2 Hemminki E, Sihvo S. A review of postmenopausal hormone therapy recommendations: potential for selection bias. Obstet Gynecol 1993;82:1021-8.

3 Dillner L. Controversy rages over new contraceptive data. BMF 1995;311:1117-8.

4 Rimpelä A, Rimpelä M, Kosunen E. Use of oral contraceptives by adolescents and its forms of contraception. The results of this review have put one piece of the family planning puzzle into place, but many other pieces remain missing.

ELINA HEMMINKI

Research professor

National Research and Development Centre for Welfare and Health,

Health Service Research Unit,

00531 Helsinki,

Finland

consequences in Finland 1981-91. BMF 1992;305:1053-7.

5 Gissler M, Rasimus A, Ritvanen A, Toukomaa H. Reproduction and its trends. SVT Health 1996;2:19.

6 Ranstam J, Olsson H. Oral contraceptive use among young women in southern Sweden. I Epidemiol Community Health 1993;47:32-5.

$7 \mathrm{McPherson} \mathrm{K}$. Breast cancer and hormonal supplements in postmenopausal women. BMF 1995;311:699-700.

\section{Guidelines for managing raised blood pressure}

\section{Evidence based or evidence burdened?}

Randomised controlled trials have consistently shown that lowering blood pressure by about $10-12 \mathrm{~mm} \mathrm{Hg}$ systolic and 5-6 $\mathrm{mm} \mathrm{Hg}$ diastolic reduces the relative risk of stroke by about $40 \%$ and of coronary disease by about $15 \%$. $^{1}$ This relative reduction in risk is similar whatever the blood pressure before treatment and the absolute risk of cardiovascular disease. Moreover, the reduction in risk occurs surprisingly quickly; the average time from the start of treatment to a significant impact on major cardiovascular outcomes is only about two and a half years.

This convincing evidence of a large and rapid relative benefit from treatment has led national and international bodies to recommend that a substantial proportion of adults be considered for long term drug treatment. In 1993 at least one international and three national guidelines on managing raised blood pressure were published. ${ }^{2-5}$ All were based on the same data, yet when Fahey and Peters (p 93) applied the various guidelines to a typical population of patients treated for hypertension in British general practice, only about a third of the patients met the treatment criteria of all four guidelines. ${ }^{6}$

The authors of all of these guidelines (including one of us, who played a role in the New Zealand guidelines) did their best to make them evidence based. However, we would suggest that the guidelines overemphasise clinically inappropriate relative measures of the benefits of treating raised blood pressure.

The movement towards evidence based medicine was in part stimulated by the observation of substantial variations in medical practice; Fahey and Peters have now shown similar variations in current guidelines for managing raised blood pressure. Evidence based medicine is "the conscientious, explicit, and judicious use of current best evidence in making decisions about the care of individual patients"7 and involves integrating the best external evidence with individual clinical expertise. The best evidence is defined as clinically relevant research, "especially patient centred clinical research into the accuracy and precision of diagnostic tests, the power of prognostic markers, and the efficacy and safety of therapeutic, rehabilitative, and preventive regimens."7 Evidence based decisions should also be mindful of competing claims on resources. We would suggest that guidelines should provide the necessary evidence based data to empower clinicians to make informed decisions. One of the most helpful tools that integrates the evidence with clinical expertise is the use of "numbers needed to treat to prevent an unwanted event or cause an unwanted side effect." 9

Calculating the number needed to treat requires the integration of external evidence (on the accuracy of diagnosis, the absolute risk before treatment, and the relative benefit of intervention) with clinical expertise in the form of judgment on the applicability of the external evidence to specific patients. We think that clinicians practising evidence based medicine should also explicitly consider the resource implications of their decisions (the cost effectiveness) at least at the level of their individual practice.

All the guidelines referred to above deal appropriately with the evidence from randomised controlled trials by examining overviews of the data, which have generally provided estimates of relative risks and benefits of treatment. ${ }^{1{ }^{10}}$ However, we suggest that, for them to guide evidence based clinical care, they need to include information on the absolute risks and benefits of interventions, as recently reported by Mulrow et al. ${ }^{11}$ Although all the guidelines acknowledge the importance of the pretreatment prognosis in determining the absolute benefits of treatment, only one of the guidelines provides the necessary information to enable clinicians to estimate the expected event rate for patients and the appropriate numbers needed to treat. ${ }^{2}$ Moreover, although all of the guidelines mentioned the importance of cost effectiveness, none provided doctors with the information needed to estimate the absolute (rather than relative) cost effectiveness of a treatment. Given that the absolute benefits and risks of treatment (however weighted by the values and resources of patients, practitioners, or payers) are crucial for making informed decisions, ${ }^{12}$ we suggest that the current guidelines have failed to provide all the evidence necessary for clinical decision making.

All four guidelines suggest threshold levels of diastolic blood pressure above which treatment should be started in all middle aged patients if the levels are sustained after nonpharmacological treatment. Three different threshold levels are recommended: $90 \mathrm{~mm} \mathrm{Hg}^{3} 95 \mathrm{~mm} \mathrm{Hg}^{5}$ and $100 \mathrm{~mm} \mathrm{Hg}{ }^{24}$ Three of the guidelines do not provide any explicit information, such as the number needed to treat or its precursors, to justify the threshold levels for treatment.

If we combine estimates of prognosis, based on data from cohort studies, ${ }^{13}{ }^{14}$ with estimates of the relative benefits of a reduction in diastolic blood pressure of 5-6 $\mathrm{mm} \mathrm{Hg}$, based on data from trials, ${ }^{1}$ it is possible to calculate numbers needed to treat that are relevant to individual patients. For example, for a 60 year old male smoker with a pretreatment diastolic blood pressure of $90 \mathrm{~mm} \mathrm{Hg}$, a ratio of total cholesterol to high density lipoprotein cholesterol of 6.5, and a normal electrocardiogram, the number needed to treat for one year (to prevent one major cardiovascular event) would be 75 . In comparison, it would be 320 for a non-smoking 50 year old woman with a considerably higher diastolic blood pressure (100 $\mathrm{mm} \mathrm{Hg}$ ), a 